\title{
Study and Analysis On Strengthening Hydrodynamic of Guazhuhu Lake Based on 2D-Numerical Simulation
}

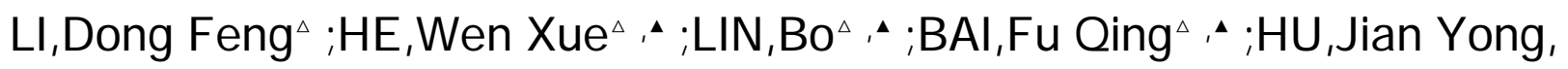 \\ NIE,Hui;YE,Zhou;J IANG ,Li J ie;XIE ,Feng
}

School of Water Resources and Environmental Engineering, Zhejiang University of Water Resources and Electric Power, Hangzhou, 310018, China;

$\triangle$ Co-first authors, all authors Contributed to this work equally

\begin{abstract}
ACo-coresponding authors: HE,WenXue,hewx@zjweu.edu.cn;LIN,Bo,linbo@zjweu.edu.cn; BAI,FuQing,baifq@zjweu.edu.cn, HU,JianYong,hujy@zjweu.edu.cn, NIE,Hui,niehui@zjweu.edu.cn
\end{abstract}

Keywords: Hydrodynamic;2D-numerical simulation; Diversion works; Guazhuhu Lake.

Abstract:In order to study the project measures for enhancing the hydrodynamic of Guazhuhu lake, the selected range included diversion works and lake surroundings. A two-dimensional mathematical model numerical simulation method was used to calculate the hydrodynamic factors, such as flow velocity and water level, the hydrodynamic characteristics of the lake waters was analyzed and three low velocity zones existing in the lake was disclosed. On the basis of above analysis of the existing low velocity properties, three water streamlining control and diversion works were designed by the theory of flow separation and vortex and flow control. The results of hydrodynamic calculation indicate that the water flow into these low velocity zones and velocity increases by the action of laid streamline guiding and control buildings, and there is no obvious vortex at the tail of flow around works, the flow resistance and head loss are also decreased.

\section{Introduction}

Guazhuhu lake is located in Keqiao District of Shaoxing, northeast of Keqiao China Textile City. North and South are about $2 \mathrm{~km}$, East and West are about $1 \mathrm{~km}$, and the water surface area is about 1.5 $\mathrm{km} 2$. The lake is the third largest lake in Shaoxing plain. The beautiful environment brings great social benefits to the city. However, because of the flat topography of the lake and its adjacent plain river network, the water movement of the lake is slow, the exchange of water is small, the self purification capacity is poor, and the eutrophication of the water body occurs frequently. Eutrophication of water bodies directly affects the sustainable development of economy and society in the lake area.

In order to prevent the lake eutrophication, in addition to the use of physical methods, chemical methods and biological methods, the water diversion from the outside region and speed up hydrodynamic is one of the important measures.

\section{Two Dimensioanl Finite Element Numerical Model}

The Governing Equations and Deterministic Conditions

The equations of continuity:

$$
\frac{\partial Z}{\partial t}+\frac{\partial(H U)}{\partial x}+\frac{\partial(H V)}{\partial y}=0
$$

The equations of motion:

$$
\frac{\partial U}{\partial t}+U \frac{\partial U}{\partial x}+V \frac{\partial U}{\partial y}+g \frac{\partial Z}{\partial x}+\frac{g n^{2} U \sqrt{U^{2}+V^{2}}}{H^{4 / 3}}-f V-\varepsilon\left(\frac{\partial^{2} U}{\partial x^{2}}+\frac{\partial^{2} U}{\partial y^{2}}\right)=0
$$




$$
\frac{\partial V}{\partial t}+U \frac{\partial V}{\partial x}+V \frac{\partial V}{\partial y}+g \frac{\partial Z}{\partial y}+\frac{g n^{2} V \sqrt{U^{2}+V^{2}}}{H^{4 / 3}}+f U-\varepsilon\left(\frac{\partial^{2} V}{\partial x^{2}}+\frac{\partial^{2} V}{\partial y^{2}}\right)=0
$$

in which, $\mathrm{U}$ and $\mathrm{V}$ is flow velocity; $\mathrm{H}$ is water depth; $\mathrm{Z}$ is elevation of water surface level ; $\mathrm{C}$ is Checy's coefficient and $C=\frac{1}{n} R^{1 / 6}$

Boundary conditions

The outlet and open boundary condition: water level process is given.

At the entrence, flow discharge is given, such as $Q(x, y, t)=Q_{0}(x, y, t), Q_{0}(x, y, t)$ is the discharge process.

For the closed and rigid boundary the velocity is zero, ie: $\left.u_{n}\right|_{\Gamma_{2}}=\left.v_{n}\right|_{\Gamma_{2}}=0$ in which, $\Gamma_{2}$

expresses closed boundary; $\mathrm{n}$ is normal unit vector.

\section{Initial conditions}

The initial water level and the initial velocity are given by measured tidal level or given value zero, initial conditions does not affect the precision of computed result.

\section{The establish of finite element model}

The finite element method is employed to solve the equations. this model, Triangle grids are selected to disperse calculation zones.

\section{Verification of the model}

Verification of the model is shown in Reference.

\section{Numerical Simulation of Lake Hydrodynamic Characteristics}

There are a few rivers around the Guazhuhu lake, the water flow direction can be opposite with time same,In other words, the water can flow into lake in one time, and can flow out the lake in the other time. This inflow and outflow makes the lake on the complex hydrodynamics, there are three low velocity regions because of the lake boundary and river location. The velocity vector field and the low velocity region of the lake are shown in Figure 1, where the velocity is large, the arrow is long, otherwise, the arrow is short. After flow stability, the contours of flow velocity at the same time are shown in Figure 2.

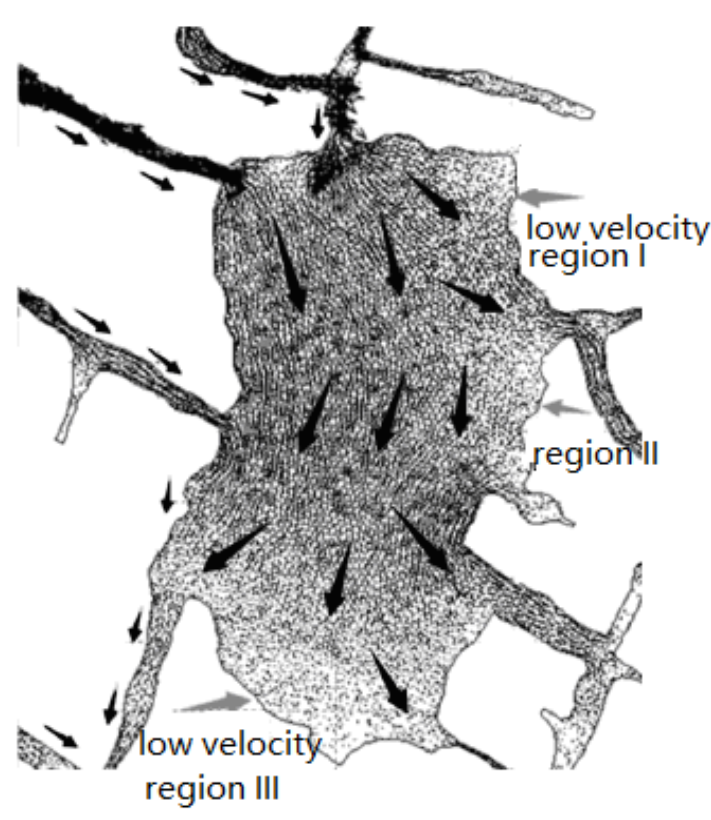

Figure 1 Velocity vector field

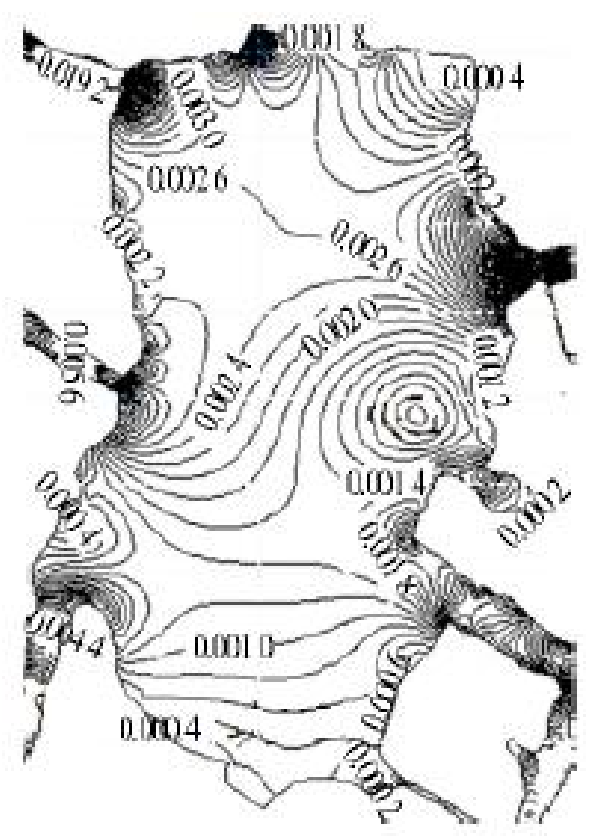

Figure 2 Velocity contour 


\section{Streamline Design Analysis of Water Flow Diversion Project in Low Flow Area}

The design of diversion project, on the one hand, the works can diverse water to the low velocity region; on the other hand, it can diverse enough water, and have less resistance, low energy loss, flow does not produce eddy current and circulation. By using the separation flow and vortex control theory and the streamline design method of the control and guidance engineering. Three different shapes of control engineering are designed in the three low velocity zones. The preliminary design is shown in Figure 3. This design also takes into account the needs of landscape function, so the circular control engineering is also the viewing platform of the lake in addition to the control flow.
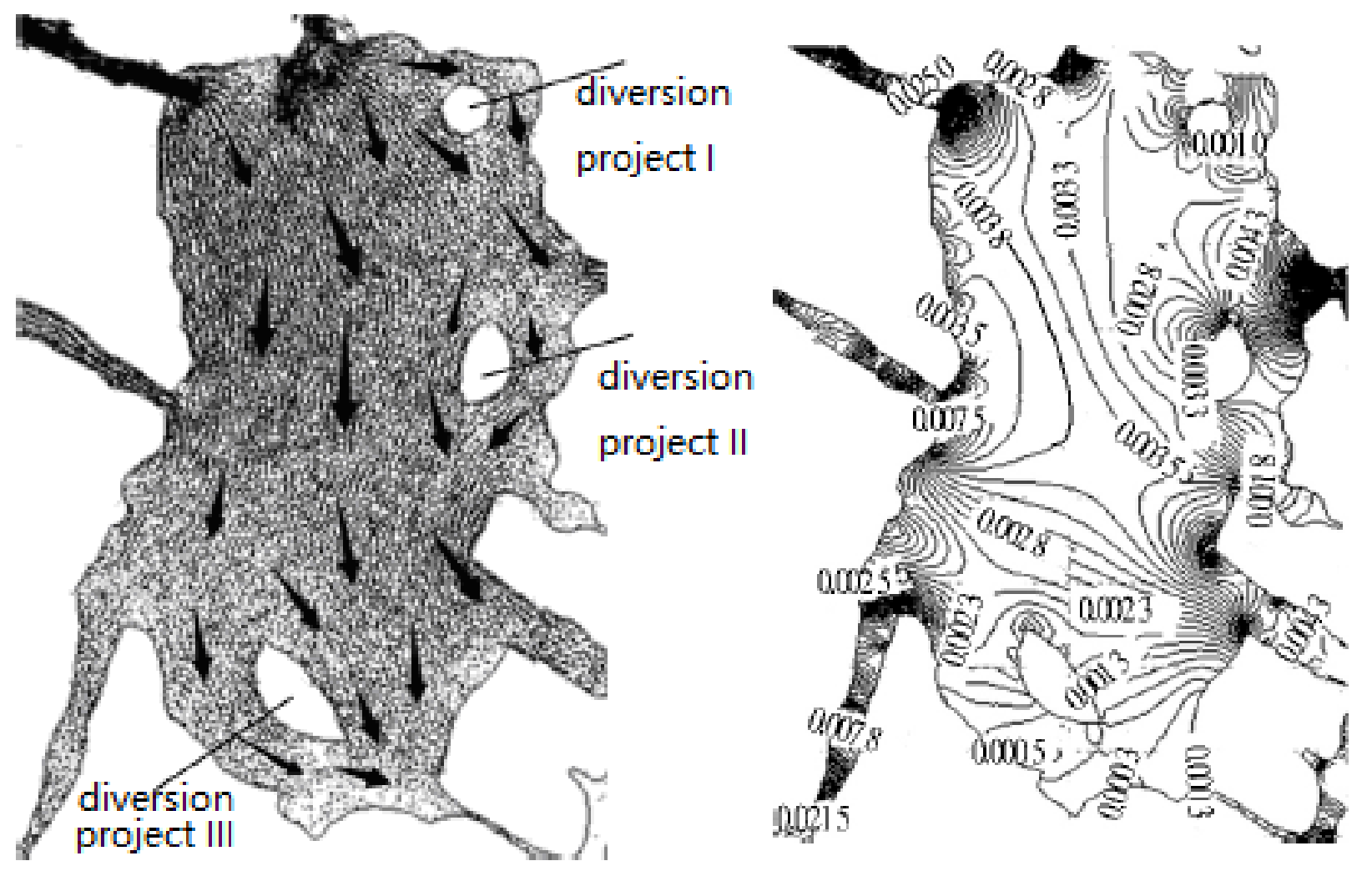

Figure3 streamlined works and velocity field Figure4 velocity contour streamlined shapes works

\section{Diversion and Strength Flow Analysis of Lake}

Figure 3 is also the velocity vector field of with three designed diversion works, Figure 4 is the velocity contour.The two Figures Indicates that the quantity of water flow to the low velocity region, and veocity increased obviously, the range of the low flow decreased,On the one hand, through the diversion of the control project, On the other hand, because the three works are streamlined, there is no obvious eddy current at the rear of the flow. The flow resistance decreases and the energy loss decreases, which is beneficial to the increase of flow velocity and the enhancement of water flow. Figure 4 shows that at low flow region I, the flow velocity at the typical position point increased from $0.001 \mathrm{~m} / \mathrm{s}$ to $0.002 \mathrm{~m} / \mathrm{s}$, and at some locations the flow rate increased more; At low flow region II, the flow velocity at the typical position point increased from $0.002 \mathrm{~m} / \mathrm{s}$ to $0.0045 \mathrm{~m} / \mathrm{s}$ and increased to 2.25 times as much; At low flow region III, the velocity of typical position points increased from $0.001 \mathrm{~m} / \mathrm{s}$ to $0.003 \mathrm{~m} / \mathrm{s}$, and the flow velocity increased to 3 times.

It can be indicated that the water flow in the low flow rate area increases obviously, and the water flow is accelerated obviously. The control and diversion project speeds up the replacement of wastewater with clean water, and reduces the probability of eutrophication in a certain extent. 


\section{Conclusions}

A two-dimensional hydrodynamic numerical model of river network and lake is used to calculate the flow of the lake. Because the time of the river network water flow into the lake is different, the interaction of the water flow in each direction makes the water flow appear complex hydrodynamic characteristics. Three low velocity regions in the Lake are revealed. Three streamlined water flow control and diversion works are designed. The quantitative analysis of water diversion and control works shows that after setting up the water control project, the velocity of the low velocity region increases to 2 3 times, and the flow of the lake water is obviously accelerated.

\section{Acknowledgements}

This work was financially supported by the Project supported by the National Key Research and Development Program of China (No.2016YFC0402502);Zhejiang Natural Science Foundation(M503254); the Water Resources bureau of Zhejiang Province (RC11092011);Zhejiang provincial Education bureau key projects (No.Z200909405), the Zhejiang provincial Education Science Plan Office Project[(2009),No.SCG220]

*All authors contributed equally to this work.

\section{References}

[1] LI Dongfeng, Zhang Hongwu, Zhong Deyu, et al. "Numerical simulation and analysis on tidal current and sediment silting process in Yellow River estuary", Journal of Hydraulic Engineering, 2004(11) .

[2] Li Dongfeng, Zou Bing, Zhang Hongwu, et al. "Two Dimensional Analysis of Tide Influence upon Estuary and Riverway" , Journal of Zhejiang Water Conservancy and Hydropower College,2006(1) .

[3] Zou Bing, Li Dongfeng, Zhang Hongwu, et al. "Effect Analysis of Building Bridges on River Hydraulic Dynamics Circumstances by 2D Numerical Method", Journal of Hangzhou Dianzi University, 2007(3).

[4] Lu hao, Gao Dongguang, "Bridge River Hydraulics" ,China Communications Press, 1996

[5] J.N. Bradley, Zheng Huaqian, "Bridge and River Hydraulic", China Communications Press, 1980

[6] Li Dongfeng, Zhang Hongwu, Zhang Junhua, et al. "Finite Element Method of Yellow River and Sediment Movement", Journal of Sediment Research, 1999(4)(in Chinese).

[7] Li Dongfeng, ZHANG Hongwu, ZHONG Deyu, et al. "2-D mathematical model for flow and sediment transport in the estuary of Yellow River", Journal of Hydraulic Engineering, 2004(6), pp $1-7$.

[8] Li Dongfeng, Zhang Jiyuan, Chen Bin, Flood influence evaluation of Chuanbuton bridge in the Haoxi River, Report of ZJWCHC,2009.

[9] Li Dongfeng, ZHANG Hongwu. Numerical Simulation Analysis of Bridge Construction Flood Flow Effect Based on Two Dimensional Finite Element Model [C], Proceedings of of 2010 International Conference on Modern Hydraulic Engineering,2010,xi'an,China.295-298 [10] Li Dongfeng, ZHANG Hongwu. Dammed Water Level Comparison of Widening Bridge Piers Based on 2D FEM Numerical Simulation and Empirical Formula [C], Proceedings of of 2010 International Conference on Modern Hydraulic Engineering,2010,xi' an,China.255-258

[11]Yang Fixiang,Pan Jie,Li Dongfeng,Zhang Hongwu. River Networks Fluidity Classification Analysis based on Two Dimensional Numerical Model and Hydraulic Characteristic.Proceedings of the 5th International Yellow River Form on Ensuring Water Right of the River' s Demand and Healthy River Basin Maintenance,Volume V,pp244-252, Yellow River Conservancy Press

[12]Yang Fixiang,Pan Jie,Li Dongfeng,Zhang Hongwu. Effect Research of River Networks Water Fluidity Enhancing Engineering Measures based on the 2D Numerical Model. Proceedings of the 5th 
International Yellow River Form on Ensuring Water Right of the River' s Demand and Healthy River Basin Maintenance, Volume V,pp302-310

[12]Zhang Huaxing,Wang Lianhua,Li Dongfeng,Zhang Hongwu. Banked-up Water Analysis of Bridge Building Two Dimensional Finite Element Numerical Simulation. Proceedings of the 5th International Yellow River Form on Ensuring Water Right of the River's Demand and Healthy River Basin Maintenance, Vol.IV,pp432-437. 